\title{
High-Speed and Non-Volatile Nano Electro-Mechanical Memory Incorporating Si Quantum Dots
}

\author{
Y. Tsuchiya ${ }^{*}$, K. Takai ${ }^{*}$ N. Momo*, T. Nagami*, \\ S. Yamaguchi ${ }^{\dagger}$, T. Shimada ${ }^{\dagger}$, H. Mizuta ${ }^{\natural}$, and S. Oda ${ }^{*}$ ฯ \\ "Quantum Nanoelectronics Research Center, Tokyo Institute of Technology, Meguro-ku, Tokyo 152-8552, Japan \\ ${ }^{\gamma}$ Central Research Laboratory, Hitachi Ltd., Kokubunji-shi, Tokyo 185-8601, Japan \\ "Department of Physical Electronics, Tokyo Institute of Technology, Meguro-ku, Tokyo 152-8552, Japan
}

\begin{abstract}
Basic device characteristics were investigated for a new high-speed and non-volatile nano electro-mechanical systems (NEMS) memory device with nanocrystalline silicon (nc-Si) dots embedded in its movable floating gate beam. Over $1 \mathrm{GHz}$ operation is possible due to the size reduction of the NEMS. From a simulation of mechanical properties of the movable floating gate beam, advantage of using the nc-Si dots array was shown for low power operation. The mechanical bistability of the fabricated $\mathrm{SiO}_{2}$ beam was clearly observed in both experimental and simulation studies.
\end{abstract}

\section{INTRODUCTION}

Nano Electro-Mechanical Systems (NEMS) have a possibility of high-speed operation in the $\mathrm{GHz}$ regime since the characteristic frequencies are expected to increase with decreasing their dimensions [1]. We proposed a new non-volatile memory concept based on bistable operation of the NEMS structure combined with nanocrystalline-Si (nc-Si) dots [2]. In this paper, we study basic device characteristics which are essential for operating our NEMS memory device.

\section{OPERATION PRINCIPLE}

Schematics of the NEMS memory device are shown in Fig. 1. Our new memory features a mechanically bistable floating gate beam, which incorporates the nc-Si dots as single-electron charge storage. The beam is suspended in the cavity under the gate electrode and moves via electrostatic interactions between the gate electrode and the charge in the nc-Si dots. Positional displacement of the beam is sensed via a change in the drain current of the MOSFET underneath.

From a mechanical analysis assuming the maximum central displacement of $50 \mathrm{~nm}$, the switching speed between two stable states was estimated to be $\sim 0.5 \mathrm{~ns}$ for a $\mathrm{SiO}_{2}$ beam with the dimension of $1.0 \times 1.0 \times 0.1 \mu \mathrm{m}^{3}$. By optimizing both the beam structure and stored charge amount, we may build an extremely fast and non-volatile memory.

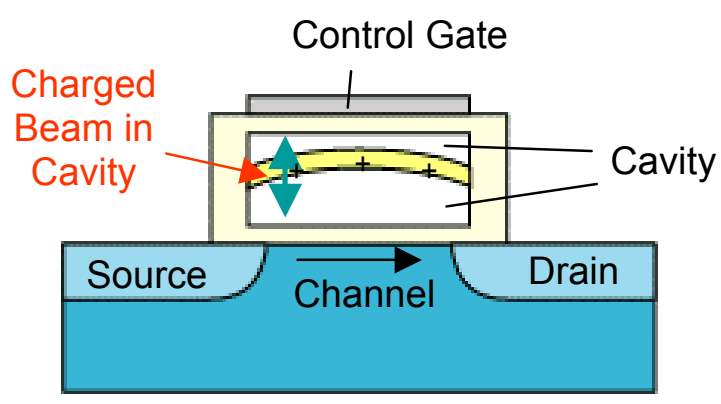

FIGURE 1. A schematic of a NEMS memory device

\section{MECHANICAL PROPERTIES}

Mechanical properties of the beam were investigated using a 3D finite element simulation [3]. We compared a nc-Si beam where a $2 \mathrm{D}$ nc-Si dot array was embedded inside a $\mathrm{SiO}_{2}$ film and a simple poly-Si beam where a thin poly-Si sheet was placed between two $\mathrm{SiO}_{2}$ layers. Calculated results under a constant uniaxial pressure are shown in Fig. 2. The large displacement was observed around at the center of the beams as shown in the rotated view. Note that the width of dark area around the center of the nc-Si beam is larger than that of the poly-Si beam. This indicates that a larger displacement is achievable with 
the nc-Si beam when an electric field is applied via the gate bias. Therefore, the nc-Si beam enables highlycontrolled charge storage and is suitable for low power operation.

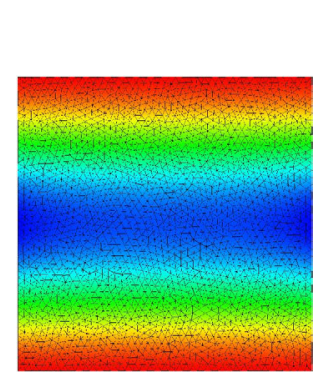

(a) nc-Si model
Along $Z$ axis

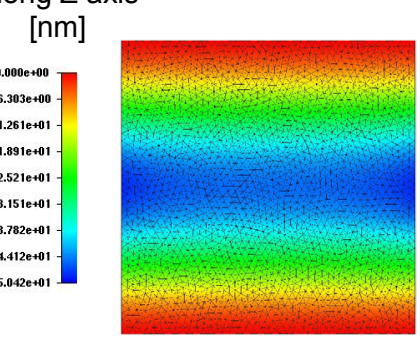

(b) Poly-Si model

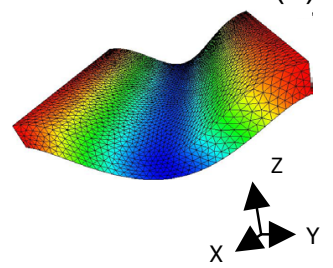

(c) Rotated view (nc-Si model)

FIGURE 2. Beam deformation simulated for two different beam structures under uniaxial pressure; Top views of (a) nc-Si beam, and (b) poly-Si beam, and rotated view of the nc-Si beam (c)

\section{BISTABILITY OF BEAM}

Bistability of the beam is a key feature for a nonvolatile memory application in this device. Figure 3(a) shows a single layer beam structure for testing its mechanical properties. After the undercut of the sacrificed Si layer underneath the beam, the most of the beams bent upward naturally as shown in Fig. 3(b), which is considered as a result of release of mechanical stress stored in $\mathrm{Si} / \mathrm{SiO}_{2}$ interface during thermal oxidation. Note that in the same test chip, several downward-bent beams were also observed as shown in Fig. 3(c). These observations strongly suggest that the beams fabricated by this method have bistable nature. This bistability was also demonstrated by using the numerical simulation which takes account of the nonlinear dependence of the beam deformation on the applied force.

\section{SUMMARY}

We have investigated basic device characteristics of the non-volatile NEMS memory device with the ncSi dots embedded in its movable floating gate beam. We found that the beam with the embedded nc-Si dots array is suitable for the movable floating gate beam to achieve low power operation. The mechanical bistability was clearly observed for the fabricated $\mathrm{SiO}_{2}$ beam.
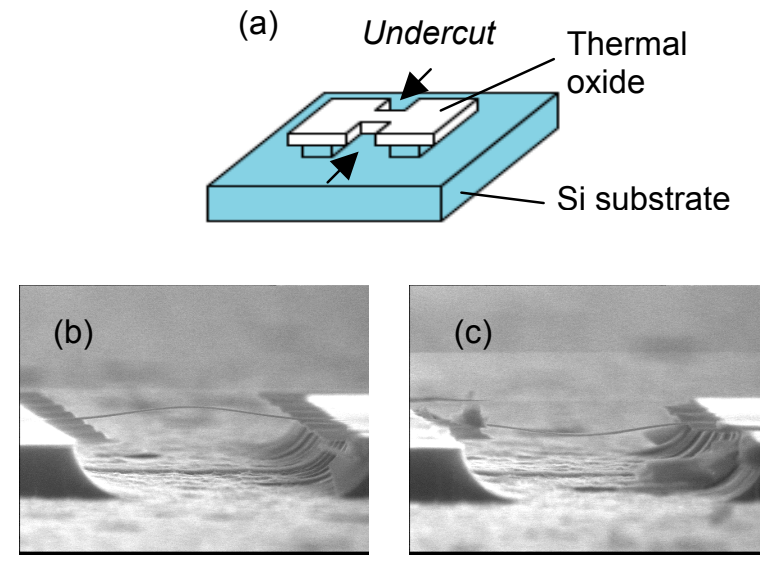

FIGURE 3. (a) A schematic illustration of the fabrication of a single layer test structure. (b) Upward-bent and (c) downward-bent $\mathrm{SiO}_{2}$ beam after undercut process.

\section{ACKNOWLEDGMENTS}

The authors are grateful to Prof. Y. Higo, Prof. K. Takashima, and Dr. S. Koyama for discussion and helpful comments. This work has been supported by CREST program of the Japan Science and Technology Agency, and IRCP of the Japan Society for the Promotion of Science.

\section{REFERENCES}

1. Huang, X. M. H., Zorman, C. A., Mehregany, M., and Roukes, M. L., Nature 421, 496 (2003).

2. Tsuchiya, Y., Takai, K., Momo, N., Yamaguchi, S., Shimada, T., Koyama, S., Takashima, K., Higo, Y., Mizuta, H., and Oda, S., in 2004 Silicon Nanoelectronics Workshop, June 13-14, 2004, Hawaii, USA

2. http://www.j-insight.com/ \& http://adventure.q.t.utokyo.ac.jp/ 